\title{
Apendicitis Aguda
}

\author{
Acute Appendicitis \\ Miguel David Flores-Mena,FACS. ${ }^{1}$
}

La Apendicitis Aguda es un proceso inflamatorio infeccioso localizado que es la causa más frecuente de abdomen agudo que requiere Cirugía de Emergencia. Su mayor incidencia es entre los 15 y 30 años. Aproximadamente 1 de cada 15 a 20 personas desarrolla apendicitis aguda durante su vida ${ }^{(1,2)}$.

Luego de casi un siglo de las contribuciones de Reginald Fitz, Charles McBurney y John Murphy hay un cambio radical en el tratamiento de la apendicitis cuando en 1983 Kurt Semm, Ginecólogo alemán realiza la primera apendicetomía laparoscópica y luego se adopta este procedimiento como una excelente alternativa ${ }^{(3,4)}$.

\section{Fisiopatología}

La apendicitis aguda se inicia con un fenómeno obstructivo por fecalitos o hipertrofia linfoide, aumento de presión intraluminal y disminución del drenaje linfático (fase catarral), más aumento de presión y obstrucción venosa (fase flemonosa) y mayor aumento de presión y compromiso arterial con isquemia (fase necrótica) que lleva a la perforación y peritonitis localizada o generalizada ${ }^{(5)}$.

\section{Cuadro clínico}

La sintomatología debe servirnos para orientarnos en el diagnóstico diferencial del abdomen agudo, el dolor abdominal en cuadrante inferior derecho es infaltable y la hiporexia es muy constante. También puede verse náuseas y/o vómitos, fiebre y alteraciones evacuatorias.

Hay algunas particularidades en la presentación del dolor como: dolor referido a fosa renal derecha si la posición es retrocecal, con diarreas por irritación recto sigmoidea, con síntomas urinarios por vecindad con la vejiga, con palidez e hipotensión en embarazo ectópico roto, de corto tiempo de evolución en cuadros médicos o patología ginecológica y sin posición antálgica en urolitiasis.

En el examen clínico debe considerarse: dolor en cuadrante inferior (punto de Mc Burney) derecho con resistencia muscular y signos peritoneales como: los signos de Blumberg o Rebote, de Dunphy o de la tos, de Markle o talones al piso y de Rovsing y dolor en FID al rebote de lado izquierdo. También el tacto rectal doloroso en el lado derecho, masa o abombamiento del fondo de saco posterior ${ }^{(6,7)}$.

\section{Exámenes auxiliares}

Leucocitosis habitualmente entre 10,000 y 18,000 leucocitos por $\mathrm{mm}^{3}$. Neutrofilia o desviación izquierda. Examen de orina para descartar patología urinaria ${ }^{(2,5,6)}$.

Radiografía de abdomen simple en busca de asa centinela por íleo localizado, borramiento del psoas, imagen de coprolito. También neumoperitoneo aunque solo en perforación apendicular ${ }^{(5,6)}$.

La ecografía o ultrasonografía es un estudio simple, inocuo, no invasivo, no usa radiación y cuya única desventaja es su dependencia del operador: pero que tiene una alta sensibilidad de 75 a $90 \%$ y alta especificidad de 85 a $100 \%$. Es recomendable en niños por las dificultades diagnósticas y en mujeres en edad fértil por la amplitud de diagnósticos diferenciales de tipo ginecológico. Pese a esto no se ha podido reducir la tasa de diagnósticos inexactos ni la tasa de perforaciones.

La tomografía es un estudio complejo que eventualmente requiere el uso de un medio de contraste y conlleva la utilización de una alta dosis de radiación ionizante lo cual significa un riesgo de cáncer en niños sometidos a este estudio de $0.18 \%$, Su sensibilidad y especificidad supera discretamente a la ultrasonografía y es recomendable en obesos, ancianos y con sospecha de neoplasia y absceso $\operatorname{apendicular}^{(5,7,8)}$.

En situaciones complejas o de duda diagnóstica se puede recurrir a la laparoscopia diagnóstica que adicionalmente puede ser terapéutica. En algunos lugares se usa rutinario en la mujeres en edad fértil ${ }^{(5,7)}$.

La resonancia magnética es cada vez es más utilizada porque no usa radiación ionizante ni contraste como la tomografía. Sin embargo no tiene alcance masivo. Por este mismo motivo, se reserva para mujeres embarazadas o niños, pacientes con riesgo de nefrotoxicidad ${ }^{(8,9)}$.

Tenemos dos problemas que han perdurado: las intervenciones innecesarias y el avance hacia la perforación con

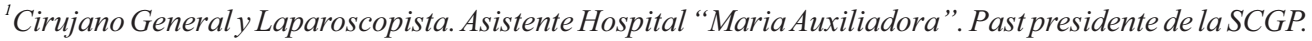


peritonitis, ya sea focal o generalizada, que puede conducir a sepsis, shock séptico, falla multiorgánica. Ambos acarrean mayores esfuerzos diagnósticos.

\section{Uso de Escalas o Scores}

Estos pacientes son abordados por un médico que no es cirujano y su disyuntiva es si referirlo a un especialista o si profundizar los medios diagnósticos. Se han desarrollado múltiples scores, haremos énfasis en el Score de Alvarado que asigna puntaje para síntomas, signos y exámenes auxiliares, que habitualmente están al alcance de cualquier centro de atención médica. (Tabla 1). de rutina y otras, hidratación, colocación de catéter venoso central, colocación de sondas nasogástrica y vesical e iniciar cobertura antibiótica según la severidad del caso ${ }^{(6)}$.

Uno de los mayores paradigmas es la inconveniencia del uso de analgésicos, actualmente luego de la decisión quirúrgica se sugiere el uso de analgésicos.

En los casos no complicados se considera tratamiento antibiótico profiláctico para cobertura para gram negativos. Tomando en cuenta a los alérgicos a betalactámicos ${ }^{(15-17)}$.

Cuando haya evidencia de perforación y peritonitis se recomienda ampliar la cobertura a gérmenes anaerobios por

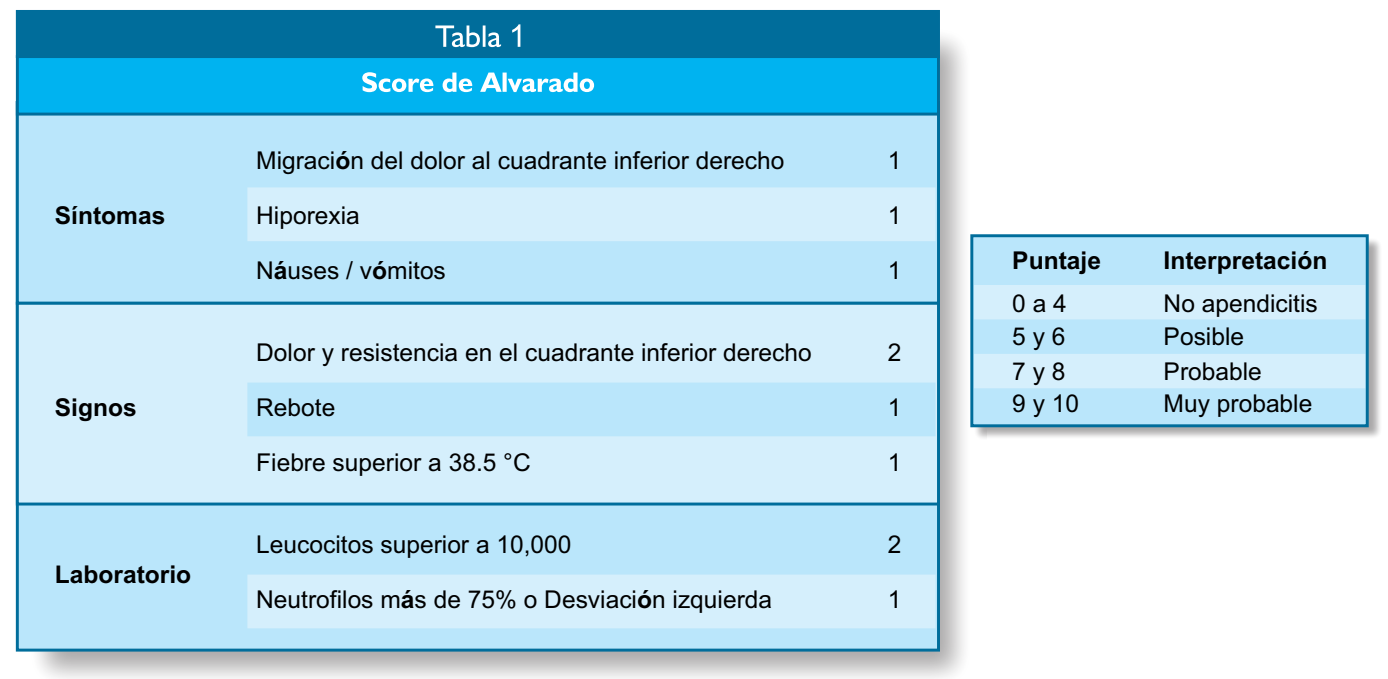

En el puntaje menor de 4 la probabilidad de apendicitis es mínima y debe continuarse evaluando clínicamente. La sensibilidad de este puntaje es de 75\%. Entre 5 y 6 puntos posible apendicitis profundizar el diagnóstico mediante métodos de imágenes y con 7 o más puntos referir para tratamiento quirúrgico ${ }^{(10-13)}$.

Existen otros scores como el de AIR (Acute Inflamatory Response) y la Escala RIPASA que han sido evaluados en cuanto a su sensibilidad con el Score de Alvarado, sin mayor ventaja ${ }^{(14)}$.

\section{Diagnóstico diferencial}

Si bien hay una gran cantidad de diagnósticos diferenciales ante un paciente con dolor abdominal en cuadrante inferior derecho la primera posibilidad es la apendicitis aguda pero los diagnósticos más frecuentes a tener en cuenta son: urolitiasis, quiste ovárico, embarazo ectópico, gastroenteritis. $(2,5,6)$

\section{Manejo preoperatorio}

En los casos de pacientes con diagnóstico de apendicitis aguda se procede a la evaluación pre operatoria lo cual se utilizan habitualmente combinaciones de antibióticos, considerando la posibilidad de monoterapias y antibiótico para infecciones para gérmenes productores de Betalactamas de expectro extendido $(\mathrm{BLEE}+)^{(15,18,19)}$

En las apendicitis agudas perforadas con peritonitis localizada o difusa se recomienda que terapia antimicrobiana no debe ser más de 5 a 7 días, a menos que no se logre un control adecuado de la fuente infecciosa ${ }^{(20)}$.

La duración del tratamiento esta en función de los hallazgos intra operatorios y la evolución clínica basada en: la normalización de la curva febril, de leucocitos y PCR, del examen abdominal y la restitución de la función intestinal. Si persiste la evidencia de infección debe considerarse: infección residual o persistente, gérmenes resistentes y causas no Infecciosas de Inflamación ${ }^{(20)}$.

\section{Tratamiento quirúrgico}

Es dependiente del diagnóstico, los casos no complicados requieren apendicetomía y cierre de piel; en los casos de peritonitis focal se plantea apendicetomía con drenaje y cierre de piel diferido y cuando se trata de peritonitis difusa apendicetomía con lavado de cavidad y cierre de piel diferido $(2,5,7)$ 
McBurney y Murphy (1889) plantearon la apendicetomía como tratamiento de elección y en 1902 se acepta como consenso este ha sido el "gold standard" para el tratamiento de esta patología, a lo largo del tiempo se ha modificado el uso de drenajes, lavado de cavidad y de antibióticos. La cirugía laparoscópica esta modificando estos $\operatorname{aspectos}^{(4)}$.

Para la decisión del tipo de cirugía se deben considerar los aspectos que favorezcan la utilización de una de las técnicas. Dentro de las ventajas de la cirugía laparoscópica están la menor intensidad del dolor post operatorio, menor estancia hospitalaria y reintegro mas rápido a sus actividades. Es ventajosa especialmente en obesos, personas mayores o con algunas comorbilidades ${ }^{(21-23)}$. En algunas circunstancias, más aún en algunas instituciones públicas se sigue dando preferencia a la cirugía abierta por la poca disponibilidad de equipos o alta demanda de pacientes. Otro factor es el entrenamiento del equipo quirúrgico, cada vez menos importante.

La cirugía laparoscópica plantea modificaciones en el uso de los drenajes. En las peritonitis focales la succión puede eliminar totalmente la secreción y prescindir del uso del drenaje; pero ante la posibilidad de persistencia de la colección sigue recomendándose el drenaje en la fosa ilíaca derecha (puerto previamente utilizado o nueva incisión). En ambas cirugías con compromiso del ciego o un muñón que no brinde confianza de su viabilidad o un neo muñón se debe utilizar el drenaje. También cuando no se identifique la base ni el apéndice cecal también procede el drenaje $\mathrm{j}^{(15,17,24)}$

En cirugía laparoscópica el lavado de cavidad requiere menor cantidad de suero fisiológico al visualizar mejor las colecciones y dirigir el lavado se esta considerando como el uso de succión sola ${ }^{(24)}$.

Se pueden presentan dos situaciones: plastrón o masa apendicular y absceso peri apendicular.

Los casos de plastrón o masa apendicular de corta evolución o con signos de abdomen agudo será sometido a apendicetomía con drenaje. Si el tiempo de enfermedad es 5 días o más, con masa abdominal palpable, sin signos peritoneales, sin fiebre y sin leucocitosis el tratamiento será conservador con hospitalización, reposo digestivo y antibióticos, evaluación ecográfica del tamaño de la masa. En 72 horas se reinicia la vía oral, terapia antibiótica oral hasta 2 semanas y alta. En mayores de 40 años se hará un estudio de imágenes o colonoscopia en busca de neoplasia el colon. No es necesaria la apendicetomía diferida por la baja incidencia de apendicitis luego en estos casos. Si no hay mejoría se considera la posibilidad de absceso peri apendicular y luego de tomografia confirmatoria se realizará su drenaje $\mathrm{e}^{(5,7,15)}$

En cirugía laparoscópica se cierran las heridas de los puertos aún en caso de perforación; pero en cirugía abierta las heridas operatorias se cierran de modo diferido. Hay algunas evidencias que abogan a favor del cierre primario. Esta conducta está respaldada principalmente por el tamaño pequeño de las heridas de los puertos y también por la baja tasa de infecciones de heridas ${ }^{(17,23)}$.

\section{Situaciones especiales}

En las gestantes la apendicitis aguda es la principal patología quirúrgica. Pueden someterse a apendicetomía laparoscópica en cualquier etapa del embarazo. Hay dificultades diagnósticas como las náuseas y vómitos habituales, hiporexia y leucocitosis y la modificación de la localización anatómica y del área de sensibilidad por el desplazamiento del ciego por el útero grávido. Se usan exámenes de imágenes especialmente la resonancia magnética, para evitar el retraso diagnóstico y la progresión de la enfermedad, con las consiguientes consecuencia de prematuridad u óbito fetal $l^{(5,17,25)}$.

Los niños y ancianos tienen baja frecuencia de apendicitis aguda, sin embargo tienen características particulares, como desarrollo lento del cuadro clínico, cuadros atípicos, baja respuesta inflamatoria y múltiples posibilidades de diagnóstico diferencial, que favorecen el retraso del diagnóstico y del tratamiento con las consecuentes complicaciones. La manera de minimizar estos efectos es mediante la aplicación temprana de métodos de diagnóstico por imágenes como ecografía y como alternativa, principalmente en niños y tomografia en ancianos en quienes es mandatorio el descarte de patología neoplásica y diverticulitis aguda. En los ancianos la cirugía laparoscópica ofrece alguna ventaja en cuanto a la evolución post operatoria $^{1,5)}$.

Los pacientes inmunocomprometidos por su condición no generan respuesta inmunitaria y no presentan leucocitosis, fiebre, defensa peritoneal por lo que se retrasa el diagnóstico. Se debe tener sospecha y utilizar tempranamente los métodos de diagnóstico como la tomografía ${ }^{(5)}$.

Durante otra intervención quirúrgica puede considerarse la apendicectomia incidental, pero por la baja incidencia de apendicitis luego de los 30 años no se recomienda su realización luego de esa edad. Tampoco en pacientes con alto riesgo de complicaciones locales como enfermedad de Crohn, post radioterapia, con prótesis vasculares, inmunosuprimidos. En cirugía laparoscópica debe considerarse como argumento en contra la posibilidad de conversión durante el procedimiento "incidental". Se recomienda en personas con dificultades para acceder a atención médica o pacientes de riesgo para evitar la posibilidad de un nuevo acto quirúrgico ${ }^{(5,6)}$.

Cuando la apendicetomía muestra un órgano macroscópicamente normal puede tratarse de cambios aún no perceptibles o determinados solo por el estudio anátomo patológico (SAS sin alteraciones significativas) o la existencia de otra patología. En caso de encontrarse alguna patología se procede a su solución y si no la hay se lleva a cabo la apendicetomía ${ }^{(5,17)}$.

\section{Complicaciones}

Las complicaciones pueden ser tempranas o tardías y su severidad y frecuencia dependen de los hallazgos intra 
operatorios. La perforación y el desarrollo de peritonitis cambian radicalmente su frecuencia de algunas de ellas. (Tabla 2).
Las infecciones de sitio operatorio (ISO) superficiales son más frecuentes en cirugía abierta y las profundas son más frecuentes en cirugía laparoscópica.

\begin{tabular}{|l|l|}
\hline \multicolumn{2}{|c|}{ Tabla 2} \\
\hline Complicaciones tempranas & \multicolumn{1}{c|}{ Complicaciones tempranas } \\
Hemorragia apendicular & Sindrome adherencial \\
Hemotama de pared & Obstrución intestinal \\
Infección del sitio operatorio (ISO) & Eventración \\
Formación de abscesos & Infertilidad por adherencias tubarias \\
Dehiscencia del muñón & \\
Fistula cecocutánea & \\
Evisceración &
\end{tabular}

La hemorragia apendicular y el hematoma de pared no están relacionadas a la apendicitis sino a la técnica operatoria, pero las demás tienen relación directa con la presencia y la magnitud de isquemia y viabilidad, la perforación y la peritonitis.
La invasión de la cavidad peritoneal puede producir tardíamente adherencias, más aún en peritonitis produciendo cuadros de síndrome adherencial y hasta obstrucción intestinal. Si el síndrome adherencial compromete las trompas uterinas a largo plazo se puede producir infertilidad $\underset{(2,6,7)}{\operatorname{trom}}$
Referencias bibliográficas

1.Townsend CM, Beauchamp RD, Evers BM, Mattox KL, y cols. Sabiston. Tratado de Cirugía. Cap. 51. Apéndice. 19a Edición 2013.

2. Morgan J, Walker H, Viggars A. Surgery on the move. CRC Press. 2014:153-158.

3.Pino W. Apendicitis Aguda. www.academia.edu

4. Mc Burney Ch. Experience with early operative interference in cases of disease of the vermiform appendix. New York Medicine Journal. 1989;50:676-684.

5. Townsend C, Beauchamp RD, Evers BM, Mattox K. Sabiston. Tratado de Cirugía. Cap. 50. Apéndice. Elsevier 20a Edicion. 2017;1296-1311.

6. Washington University. Manual Washington de Cirugía. Marban Libros. $3^{\text {a }}$ edición. 2005.

7. Giménez M. Fundamentos para la práctica clínico-quirúrgica. Apendicitis Aguda. Ed. Médica Panamericana 2014;53:583593.

8. Arévalo Espejo O, Moreno Mejía ME, Ulloa Guerrero L. Apendicitis aguda: Hallazgos radiológicos y enfoque actual de las imágenes diagnósticas. Rev. Colomb. Radio. 2014;25(1): 3877-3888.

9. Gerhard F, Soffia Pablo, Silva C, Labra A. Utilidad de la resonancia magnética para el diagnóstico de apendicitis aguda en pacientes embarazadas. http://contactocientifico.alemana. cl/ojs/index.php/cc/article/view/351/330

10. The Cochrane Library. The Alvarado Score for predicting acute Appendicitis: A sistematic Review. BMC Medicine. 2011.

11.Andersson M, Andersson RE. The Appendicitis Inflammatory Response Score: A Tool for the Diagnosis of Acute Appendicitis that Outperforms the Alvarado Score. World J Surg. 2008;32:1843-1849.
12. Quesada Suárez L, Ival Pelayo M, González Meriño CL. La escala de Alvarado como recurso clínico para el diagnóstico de la apendicitis aguda. Rev. Cubana Cir. 2015;54(2):121-128.

13.Meléndez Flores JE, Cosio Dueñas H, Sarmiento Herrera WS. Sensibilidad y especificidad de la Escala de Alvarado en el diagnóstico de pacientes atendidos por apendicitis aguda en hospitales del Cusco. Horiz. Med. 2019;19(1):13-18.

14.Díaz-Barrientosa CZ, Aquino-González A, HerediaMontaño M, Navarro-Tovar F, Pineda-Espinosa MA, Espinosa de Santillana IA. Escala RIPASA para el diagnóstico de apendicitis aguda: comparación con la escala de Alvarado modificada. Revista de Gastroenterología de México. 2018;83(2):112-116.

15. Hospital María Auxiliadora. Departamento de Cirugía, Servicio de Cirugía General. Guía Práctica Clínica de Apendicitis Aguda. Hospital María Auxiliadora 2011.

16. American Society of Health System. Clinical Practice Guidelines for Antimicrobial Prophylaxis in Surgery. 2013.

17.EAES (European Association of Endoscopic Surgery). Diagnosis and management of acute appendicitis. EAES consensus development conference 2015. Surg Endosc (2016).

18. World Society of Emergency Surgery (WSES). WSES guidelines for management of intraabdominal infections. The management of intra-abdominal infections from a global perspective. 2017.

19.Surgical Infection Society. Revised Guidelines on the Management of Intra-Abdominal Infection. 2017.

20.Association of Medical Microbiology and Infectious Disease (AMMI) Canada. Canadian Guidelines of Intra abdominal Infections. Canadian Journal of Infectious diseases. 2010.

21.Ingraham AM, Cohen ME, Bilimoria KY, et al. Comparison of outcomes after laparoscopic versus open appendectomy for 
acute appendicitis at 222 ACS NSQIP, Surgery 2010;148:625635.

22. Society of American Gastrointestinal and Endoscopic Surgeons (SAGES). Guidelines for Laparoscopic Appendectomy. 2009.

23.World Society of Emergency Surgery WSES. Jerusalem guidelines for diagnosis and treatment of acute appendicitis.
World Journal of Emergency Surgery. 2016:11:34.

24. The Cochrane Library. Abdominal Drainage after Appendectomy for Complicated Appendicitis. 2012.

25. Society of American Gastrointestinal and Endoscopic Surgeons (SAGES). Guidelines for the Use of Laparoscopy during Pregnancy, 2017.

Contribución de autoría: Miguel David Flores-Mena ha sido el autor del estudio, contribuyendo con su concepción, búsqueda electrónica, revisión inicial, el diseño de estudio, redacción, y revisión final.

Conflicto de interés: El autor no tiene conflictos de interés con la publicación de este trabajo.

Financiamiento: Autofinanciado.

Citar como: Flores-Mena MD. Abdomen Agudo. Diagnóstico(Lima). 2020;59(4):213-217.

DOI: 10.33734/diagnostico.v59i4.257

Correspondencia: Miguel David Flores Mena. Correo electrónico: drmflores_cirugia@yahoo.com

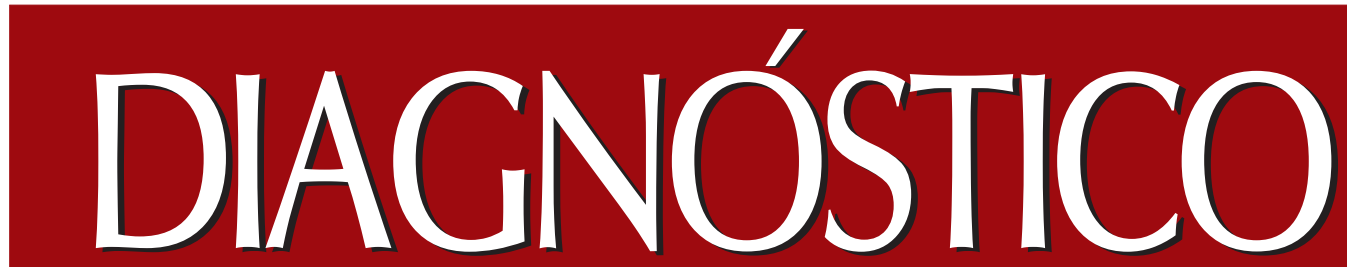

Revista Médica de la Fundación Instituto Hipólito Unanue

\section{Toda la información médica que ofrece la}
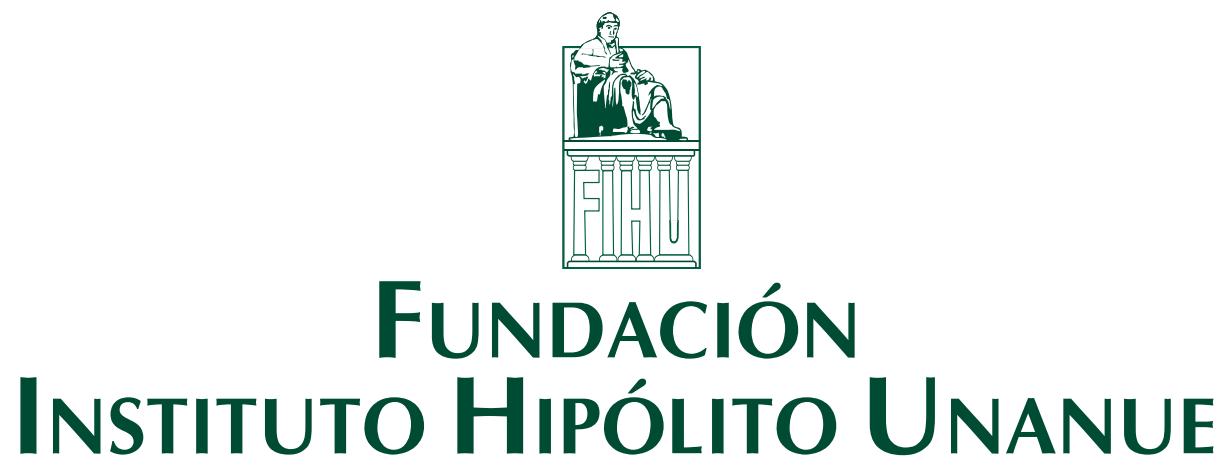

está en Internet

- Versión en línea de la revista

- Buscador Temático dentro de la revista

- Noticias Médicas

- Informaciones sobre la Fundación

- Premio Medalla de Oro Hipólito Unanue

- Premio Hipólito Unanue a los Mejores Trabajos de Investigación en las

Ciencias de la Salud

- Premio Hipólito Unanue a la Mejor Edición Científica sobre Ciencias de la Salud

- Apoyo Económico a la Investigación Científica

- Becas de Honor

-Actividades Científicas en Provincias - Cursos Multidisciplinarios

\section{www.fihu.org.pe}

